\title{
A experiência do puerpério para as famílias: revisão integrativa
}

\author{
The experience of the puerperium for the families: an integrative review \\ La experiencia del puerperio para las familias: revisión integrativa
}

Fernanda Rios da Silva

ORCID: https://orcid.org/0000-0002-6584-6651 Universidade Federal do Paraná, Brasil

E-mail: enfafernandarios@hotmail.com

Maria Ribeiro Lacerda

ORCID: https://orcid.org/0000-0002-5035-0434 Universidade Federal do Paraná, Brasil

E-mail: mrlacerda55@gmail.com

Ingrid Meireles Gomes

ORCID: https://orcid.org/0000-0002-2174-6421 Universidade Federal do Paraná, Brasil E-mail: inguide@gmail.com

Adelita Gonzalez Martinez Denipote

ORCID: https://orcid.org/0000-0003-2893-0499 Universidade Federal do Paraná, Brasil E-mail: adenurse@gmail.com

Luciana Midori Teruya

ORCID: https://orcid.org/0000-0001-9126-1158 Hospital Paulista, Brasil

E-mail: luciana.teruya@icloud.com

\begin{abstract}
Resumo
Objetivo: sistematizar as evidências disponíveis na literatura sobre a experiência do puerpério para as famílias. Metodologia: revisão integrativa nas bases: Medical Literature Analysis and Retrieval System Online, Cumulative Index to Nursing \& Allied Health Literature, Web of Science, Scopus, Scientific Electronic Library Online e ScienceDirect e na Biblioteca Virtual de Saúde. Nas bases e portal pesquisados, foram feitos cruzamentos utilizando os descritores e palavras-chaves: famílias, relacionamento familiar, relação familiar, dinâmica familiar, período pósparto, puerpério, pós-parto da mulher, habitação, domicílio, domiciliar, doméstico, apartamento, apartamentos, casa, casas, lar, lares, moradia, moradias, experência de vida, e acontecimentos que mudam a vida. Resultados: a amostra foi constituída por oito artigos que apresentaram valores culturais repassados de uma geração para outra, que influenciam a família na criação de novos membros; a melhoria das relações familiares através da prática de exercícios físicos pelas puérperas; o uso de mídias sociais e das ferramentas: Modelo Calgary de Avaliação da Família, Modelo Calgary de Intervenção Familiar e Newborn Behavioral Observation, para apoiar a prática profissional baseada em evidências. Considerações finais: as transições no ciclo da vida, embora façam parte do processo de desenvolvimento familiar, em geral são acompanhadas por conflitos marcantes. As transformações ocorridas no puerpério, especificamente, afetam a dinâmica familiar, requerendo dos profissionais de saúde, sobretudo das enfermeiras, suporte para a adaptação à nova realidade.
\end{abstract}

Palavras-chave: Mulheres; Período pós-parto; Família; Relações familiares; Enfermeiros.

\begin{abstract}
Objective: to systemize available evidence in literature on the experience of the puerperium for the families. Methodology: an integrative review in databases: Medical Literature Analysis and Retrieval System Online, Cumulative Index to Nursing \& Allied Health Literature, Web of Science, Scopus, Scientific Electronic Library Online and ScienceDirect as well as in the Virtual Health Library. In databases and researched, gates was done the crossing using the descriptors and keywords: families, family relationship, family relation, family dynamic, postpartum period, puerperium, postpartum, postpartum women, housing, domicile, domicile, domestic, apartment, apartments, house, houses, home, homes, dwelling, dwellings, life change events, experience, life. Results: the sample was constituted by eight articles that present cultural values transmitted generation by generation to other, that influence the family in the parenting of new members; the improvement of family relationship by practicing physical exercises by the women in puerperium; the use of social medias and the tools: Calgary Model of Family Assessment, Calgary Family Intervention Model and Newborn Behavioral Observation, to support the professional practice evidence based. Final considerations: the life cycle transitions, although makes part of the familiar development process, are generally accompanied by striking conflicts. The transformations occurred in puerperium, specifically,
\end{abstract}


affect the family dynamic, requiring of health professionals, mainly nurses, support for adaptation to the new reality. Keywords: Women; Postpartum period; Family; Family relations; Nurses Male.

\section{Resumen}

Objetivo: sistematizar las evidencias disponibles en la literatura referente a la experiencia del puerperio para las familias. Metodología: revisión en las bases: Medical Literature Analysis and Retrieval System Online, Cumulative Index to Nursing \& Allied Health Literature, Web of Science, Scopus, Scientific Electronic Library Online y ScienceDirect y en la Biblioteca Virtual de la Salud. En las bases y portales investigados, fueron hechos tres cruces usando los descriptores y palabras claves: familias, relación familiar, dinámica de la familia, periodo posparto, mujer posparto, vivienda, residencia, domicilio, doméstico, apartamento, apartamentos, casa, casas, hogar, hogares, vivienda, viviendas, experencia de vida, acontecimientos que cambian la vida. Resultados: la muestra fue constituida por ocho artículos que representaran valores culturales repasados de generación a generación para otra, que influencian la familia en la creación de nuevos miembros, la mejoría de las relaciones familiares por medio de la práctica de ejercicios físicos por las puérperas; el uso de redes sociales y herramientas: Modelo Calgary de Evaluación de la Familia, Modelo Calgary de Intervención Familiar y Newborn Behavioral Observation, para apoyar la práctica profesional basada en evidencias. Consideraciones finales: transiciones en el ciclo de vida, aunque hagan parte del proceso de desarrollo familiar, por lo general son acompañadas por conflictos que marcan. Las transformaciones ocurridas en el postparto, específicamente, afectan la dinámica familiar, requiriendo de los sanitarios, sobre todo de las enfermeras, soporte para adaptación a la nueva realidad.

Palabras clave: Mujeres; Periodo posparto; Familia; Relaciones familiares; Enfermeros.

\section{Introdução}

Diariamente, em torno de 830 mulheres morrem ao redor do mundo por causas evitáveis relacionadas à gestação e ao parto, sendo que ações e cuidados antes, durante e após o parto podem reduzir significativamente esta estimativa que é maior em países em desenvolvimento (Organização Pan-Americana da Saúde [OPAS] \& Organização Mundial da Saúde [OMS], 2018).

No ano 2000, por entender que a mortalidade materna corresponde a um indicador de desenvolvimento social, o Brasil e outros 190 países-membros da Organização das Nações Unidas (ONU) desenvolveram os Objetivos de Desenvolvimento do Milênio (ODM), um ambicioso conjunto de metas a serem cumpridas entre os anos 2000 e 2015, entre elas a redução do índice de mortalidade materna. Todavia, decorrido este prazo, mesmo com alguns avanços, havia um quantitativo substancial de mortes maternas em todo o mundo (Souza, 2015). Assim, um novo documento foi emitido com novas metas a serem cumpridas até 2030. Este ficou conhecido como Objetivos de Desenvolvimento Sustentável (ODS) e tem a redução da mortalidade materna como um dos principais desafios perseguidos (Roma, 2019).

Mesmo com este propósito, a realidade é que o índice de morbimortalidade materna continua elevado e, para reduzilo, é necessário o firme compromisso de governos em todo o mundo em melhorar a atenção à saúde das mulheres (OPAS \& OMS, 2018).

O Brasil, de modo específico, sobretudo a partir da implantação do Sistema Único de Saúde (SUS), tem investido na criação de normativas e programas dirigidos à saúde das mulheres, entre eles a Rede Cegonha (RC) que, por meio dos seus quatro componentes - Pré-natal; Parto e nascimento; Puerpério e Saúde da criança; e Transporte sanitário e regulação, define ações a fim de garantir o acesso e a prestação de cuidado no período perinatal (Brasil, 2011).

O puerpério, particularmente, é o período em que o organismo feminino, que passou por alterações fisiológicas e bioquímicas durante a gestação, percorre o caminho de retorno às condições pré-gravídicas (Montenegro \& Rezende, 2017). Este é subdividido em três períodos: puerpério imediato $-1^{\circ}$ ao $10^{\circ}$ dia após o parto; puerpério tardio $-11^{\circ}$ ao $45^{\circ}$ dia após o parto; e puerpério remoto - do $45^{\circ}$ dia após o parto sem previsão de término, pois seu início ocorre com a dequitação placentária, enquanto seu fim depende do tempo que o organismo de cada mulher necessitará para retornar ao completo estado não gravídico (Brasil, 2016). 
De modo geral, o puerpério costuma ser vivenciado pela mulher junto a família - a sua principal rede de apoio, no domicílio. Entende-se como família uma unidade dinâmica composta por pessoas que convivem juntas porque tem laços consanguíneos, de afetividade, interesse e/ou doação. A família tem como funções: proporcionar afeto, cuidado e apoio a seus membros, além de permitir o compartilhamento de objetivos, responsabilidades, direitos e obrigações. Assim, como instituição social básica a família é mais que algo natural ou dado com caráter biológico, ela é o produto de construções históricas e retrata as formas dos homens se organizarem coletivamente (Gomes, 2016).

Considera-se o puerpério um período desafiador à família, pois transições no ciclo de vida, mesmo fazendo parte do processo de desenvolvimento familiar, em geral provocam desconstrução, desestruturação e reconstrução dos envolvidos no que se refere aos aspectos envolvendo a dinâmica familiar (relação conjugal, desestruturação da identidade ao passar da condição de filho/filha e esposa/esposo para pai/mãe, financeira, social, entre outros), como apontado em uma clássica obra que fornece uma estrutura para a terapia familiar nas mudanças do ciclo de vida (Carter \& McGoldrick, 2011).

Na transição à maternidade, a mulher passa por intensas transformações que a levam a estabelecer novas rotinas, prioridades na vida, novos comportamentos, princípios e valores. A passagem por este processo marca e modifica a família (Demarchi, et al., 2017). O puerpério, nomeadamente, é um período de mudanças que ultrapassam a dimensão biológica da mulher, haja vista que outras áreas (conjugal, familiar, social e profissional) também são afetadas (Montenegro \& Rezende, 2017). Mesmo diante desta constatação, são poucas as investigações que dão visibilidade a este grupo social quando se trata do puerpério.

Por acreditar que explorar sobre a experiência do puerpério para as famílias é trazer a possibilidade de ampliar a compreensão sobre este tema e suscitar reflexões capazes de melhorar as práticas dos profissionais de saúde, desenvolveu-se o presente estudo cujo objetivo é: sistematizar as evidências disponíveis na literatura sobre a experiência do puerpério para as famílias.

O presente artigo faz parte da Pesquisa de Doutorado, em desenvolvimento, intitulada "A experiência do puerpério para as famílias”, da Universidade Federal do Paraná (UFPR). Sua construção teve como finalidade conhecer o estado da arte e as lacunas do conhecimento referente a temática.

\section{Metodologia}

Trata-se de uma revisão integrativa, tipo bibliográfico de estudo que possibilita o acesso ao estado atual do conhecimento acerca de um determinado fenômeno, a partir da busca, avaliação crítica e síntese das evidências disponíveis (Mendes, Silveira, \& Galvão, 2019).

As etapas percorridas para o desenvolvimento do estudo foram: 1) formulação da questão de pesquisa - utilizando o acrônimo PICo; 2) amostragem - estabelecimento de critérios de inclusão e exclusão; busca nas bases e seleção dos estudos; 3 ) extração dos dados - apreciação na íntegra dos estudos que restaram após a leitura dos títulos e resumos; composição do corpus da revisão; elaboração de dois instrumentos para recolha de informações; 4) avaliação crítica - classificação dos estudos pelo Nível de Evidência (NE); 5) análise e síntese dos resultados - coleta de informações que respondem à questão de pesquisa; e 6) apresentação da revisão - divulgação dos resultados (Paula, Padoin, \& Galvão, 2016).

Foi adotada a estratégia PICo (Lockwood, Munn, \& Porritt, 2015) para a elaboração da questão de pesquisa, na qual (P) População: famílias; (I) Interesse: experiência do puerpério; e (Co) Contexto: domicílio. Conforme essa estratégia, a pergunta de pesquisa elaborada foi: Quais as evidências disponíveis na literatura sobre a experiência do puerpério para as famílias no contexto domiciliar? 
Para responder à questão de pesquisa, acessou-se as bases de dados: Medical Literature Analysis and Retrieval System Online (Medline), Cumulative Index to Nursing \& Allied Health Literature (CINAHL), Web of Science (WoS), Scopus, Scientific Electronic Library Online (SciELO) e ScienceDirect, além da Biblioteca Virtual de Saúde (BVS), utilizando Descritores em Ciências da Saúde (DECS) e seus respectivos sinônimos combinados entre si por meio de operadores booleanos $(O R / A N D)$, com o intuito de ampliar a possibilidade de localização de estudos que respondessem à pergunta de pesquisa. As bases de dados e portal consultados, bem como suas respectivas estratégias de busca encontram-se no Quadro 1.

Quadro 1. Bases de dados e portal, consultados com suas respectivas estratégias de buscas utilizadas para levantamento.

\begin{tabular}{|c|c|}
\hline Bases de dados & Estratégia de busca \\
\hline MedLine, CINAHL, WoS, SciELO e BVS & $\begin{array}{l}\text { ("Relacionamento Familiar" OR "Relação Familiar" OR "Dynamic, Family" OR } \\
\text { "Dynamics, Family" OR "Family Dynamic" OR "Family Dynamics" OR "Family } \\
\text { Relation" OR "Family Relationship" OR "Family Relationships" OR "Relation, Family" } \\
\text { OR "Relations, Family" OR "Relationship, Family" OR "Relationships, Family") AND } \\
\text { ("Período Pós-Parto" OR "Postpartum Period" OR "Periodo Posparto" OR "Puerpério" } \\
\text { OR "Period, Postpartum" OR "Postpartum" OR "Postpartum Women" OR "Women, } \\
\text { Postpartum" OR "Puerperium") AND ("Habitação" OR "Housing" OR "Vivienda" OR } \\
\text { "Domicílio" OR "Domiciliar" OR "Apartamento" OR "Apartamentos" OR "Casas" OR } \\
\text { "Casa" OR "Lar" OR "Lares" OR "Moradia" OR "Doméstico" OR "Domestic" OR } \\
\text { "Moradias" OR "Home" OR "House") }\end{array}$ \\
\hline Scopus e ScienceDirect & $\begin{array}{l}\text { ("Life Change Events" OR "Experience, Life") AND ("Famílias" OR "Family") AND } \\
\text { ("Postpartum Period" OR "Periodo Posparto" OR "Puerpério") AND ("Domicílio" OR } \\
\text { "Home") }\end{array}$ \\
\hline
\end{tabular}

Fonte: Autores (2020).

Para corroborar a exaustão da possibilidade de busca o acesso, ocorrido no mês de julho de 2020, deu-se eu através do portal de periódicos da Coordenação de Aperfeiçoamento de Pessoal de Nível Superior (CAPES) em cobertura de Internet Protocol (IP) pertencente à Universidade Federal do Paraná (UFPR). Adotou-se o critério de seleção duplo-independente como controle de qualidade, assim, as publicações foram analisadas por duas das cinco autoras separadamente, não havendo discordâncias que não se chegasse ao consenso.

Preliminarmente, para a filtragem da busca com os recursos contidos nas bases, foram selecionados artigos com textos completos, nos idiomas português, inglês e ou espanhol, publicados entre os anos 2015 a 2020, considerando a procura pela atualidade sobre o fenômeno de interesse.

Como critérios de inclusão estabeleceu-se: artigos originais disponíveis na íntegra, em qualquer idioma, publicados nos últimos cinco anos, que apresentassem relação com o objeto de pesquisa. E como critérios de exclusão definiu-se: estudos de revisão e de caso, editoriais, resenhas, capítulos de livro, resumos publicados em anais de evento, monografias, dissertações, teses e artigos repetidos entre as bases de dados pesquisadas, sendo mantida apenas a primeira versão identificada.

Feito o levantamento nas bases, os estudos capturados foram lidos seguindo a ordem: título, resumo e na íntegra. As autoras criaram uma ficha para justificar a exclusão das produções que não atendiam aos critérios de inclusão. Este recurso facilitou a sistematização da seleção e garantiu a fidedignidade dos resultados (Paula, et al., 2016). Os estudos observados a 
partir da leitura dos títulos e resumos que atenderam aos critérios de inclusão e responderam à pergunta de pesquisa foram selecionados para a leitura na íntegra.

Os artigos selecionados foram exaustivamente lidos. As informações relevantes de cada estudo foram extraídas para dois quadros. O primeiro continha as variáveis externas: título, autores, idioma, ano de publicação, periódico e base de indexação. E o segundo as variáveis internas: título, tipo de estudo, principais resultados e a classificação do NE (Melnyk \& Fineout-Overholt, 2005; Paula, et al., 2016).

A fim de reunir o conhecimento produzido sobre o tema explorado, a análise dos dados foi feita de forma descritiva, possibilitando observar e descrever os resultados.

\section{Resultados}

Foram identificadas 157 publicações sobre o tema a partir da busca sem filtros nas bases de dados. Após filtragem, considerando os critérios de inclusão e exclusão, foram escolhidos 43 artigos para a leitura dos títulos e resumos. Destes, 12 foram lidos na íntegra, sendo a amostra final composta por oito estudos, como mostra a Figura 1.

Figura 1. Fluxograma de identificação e seleção dos estudos integrados a revisão.

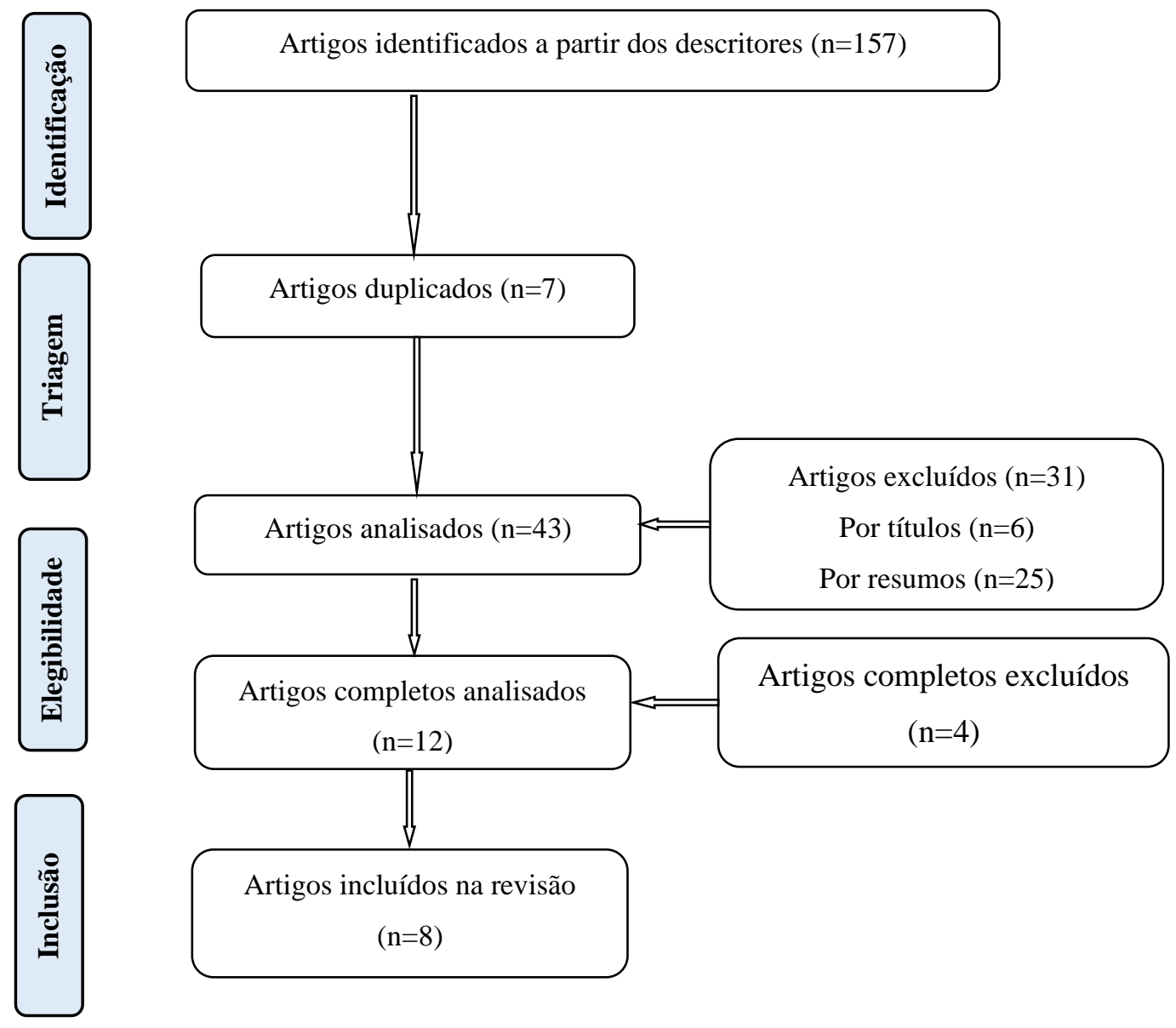

Fonte: Autores (2020).

Dos artigos que compuseram o corpus da revisão, seis foram publicados na língua inglesa (Aston et al., 2015; Asiodu, Waters, Dailey, Lee, \& Lyndon, 2015; Zeiders, Umaña-Taylor, Jahromi, \& Updegraff, 2015; Hutt, Moore, Mammen, 
\& Downs, 2017; Kristensen \& Kronborg, 2018; Cava-Tadik, Brown \& Mangelsdorf, 2020) e dois nas línguas inglesa e portuguesa (Melo, Calderon, Monteiro, \& Veríssimo, 2015; Demarchi, et al., 2017). Desta amostra, três produções estavam indexadas na base de dados Cinhal (Melo, et al., 2015; Demarchi, et al., 2017; Cava-Tadik, et al., 2020), três na BVS (Aston, et al., 2015; Hutt, et al., 2018; Kristensen \& Kronborg, 2018), uma na ScienceDirect (Asiodu, et al., 2015) e uma na Medline (Zeiders, et al., 2015).

Em relação a distribuição geográfica e temporal, quatro estudos $(50 \%)$ foram realizados nos Estados Unidos da América (EUA) (Asiodu, et al., 2015; Hutt, et al., 2017; Cava-Tadik, et al., 2020), dois (25\%) no Brasil (Melo, et al., 2015; Demarchi, et al., 2017), um (12,5\%) no Canadá (Aston, et al., 2015) e um (12,5\%) na Dinamarca (Kristensen \& Kronborg, 2018).

O ano 2015 contou com o maior número de publicações, somando um total de quatro (Aston, et al., 2015; Asiodu, et al., 2015; Zeiders, et al., 2015; Melo, et al., 2015) seguido de 2017 com duas (Demarchi, et al., 2017; Hutt, et al., 2017); e os anos 2018 (Kristensen \& Kronborg, 2018) e 2020 (Cava-Tadik, et al., 2020) com uma em cada ano, respectivamente.

Quanto ao tipo de estudo, metade das produções (50\%) seguiram a abordagem qualitativa (Aston, et al., 2015; Asiodu, et al., 2015; Demarchi, et al., 2017; Melo, et al., 2015), e a outra metade a abordagem quantitativa (Zeiders, et al., 2015; Cava-Tadik, et al., 2020). Os achados referentes a distribuição dos estudos analisados encontram-se no Quadro 2.

Quadro 2. Caracterização do corpus de análise da revisão integrativa segundo base de dados ou portal, primeiro autor, periódico, local, ano de publicação, principais resultados e NE.

\begin{tabular}{|c|c|c|c|c|}
\hline $\begin{array}{c}\text { Base ou } \\
\text { Portal }\end{array}$ & $\begin{array}{c}\text { Primeiro autor/ } \\
\text { Periódico }\end{array}$ & $\begin{array}{l}\text { Local/ Ano } \\
\text { de } \\
\text { publicação }\end{array}$ & Principais resultados & NE \\
\hline Cinhal & $\begin{array}{l}\text { Melo/ Rev } \\
\text { enferm UFPE on } \\
\text { line }\end{array}$ & Brasil/ 2015 & $\begin{array}{l}\text { As mulheres com Depressão Pós-Parto (DPP) não se sentem bem compreendidas } \\
\text { pelos parceiros (falta diálogo, carinho, amor e respeito) e acabam sofrendo desapego } \\
\text { com os filhos e déficit de interação mãe-filho, além de discórdia entre as famílias e } \\
\text { preconceito entre os irmãos. Também, tiveram relatos de paciência, tolerância, } \\
\text { gratidão, afeto, acolhimento e companhia. A família é reconhecida como essencial } \\
\text { para desfecho satisfatório do puerpério. }\end{array}$ & N6 \\
\hline $\begin{array}{l}\text { Science } \\
\text { Direct }\end{array}$ & $\begin{array}{l}\text { Asiodu/ J Obstet } \\
\text { Gynecol } \\
\text { Neonatal Nurs }\end{array}$ & EUA/ 2015 & $\begin{array}{l}\text { Aborda o uso de mídias sociais para compartilhar eventos e coletar informações } \\
\text { sobre desenvolvimento fetal, orientações sobre parto, alterações corporais, } \\
\text { parentalidade e nutrição, sobretudo alimentação infantil. Em algumas situações, ao } \\
\text { se sentirem sozinhas as puérperas recorriam às mídias sociais como forma de } \\
\text { substituir o apoio familial. Também, houve situações em que o uso das mídias } \\
\text { sociais serviu como elo entre a mulher e a família. }\end{array}$ & N6 \\
\hline Medline & $\begin{array}{l}\text { Zeiders/ } \\
\text { J Fam Psychol }\end{array}$ & EUA/ 2015 & $\begin{array}{l}\text { Demonstra que os valores do familismo das avós antes do nascimento do bebê } \\
\text { previam apoio e comunicação à criação na díade mãe-avó após o nascimento da } \\
\text { criança. Houve envolvimento intergeracional no puerpério das adolescentes com } \\
\text { repercussões positivas. }\end{array}$ & $\mathrm{N} 4$ \\
\hline BVS & $\begin{array}{l}\text { Hutt/ Research } \\
\text { Quarterly for } \\
\text { Exercise and } \\
\text { Sport }\end{array}$ & EUA/ 2017 & $\begin{array}{l}\text { Exercícios físicos tem potencial para melhorar as relações familiares em especial } \\
\text { durante o primeiro ano pós-parto, tempo necessário para os ajustes na família } \\
\text { decorrentes da chegada de um novo membro. No puerpério, o estresse conjugal } \\
\text { aumenta, especialmente para mães com mais de um filho. }\end{array}$ & N6 \\
\hline
\end{tabular}




\begin{tabular}{|c|c|c|c|c|}
\hline Cinhal & $\begin{array}{l}\text { Demarchi/ Rev } \\
\text { enferm UFPE on } \\
\text { line }\end{array}$ & Brasil/ 2017 & $\begin{array}{l}\text { A família exerce valioso papel por dispensar cuidados ao recém-nascido, outros } \\
\text { filhos e as puérperas, auxiliar em questões psicológicas como na melhoria da } \\
\text { autoestima e dar força à puérpera no enfrentamento de dificuldades. }\end{array}$ & N6 \\
\hline BVS & $\begin{array}{l}\text { Kristensen/ BMC } \\
\text { Public Health }\end{array}$ & $\begin{array}{l}\text { Dinamarca/ } \\
2018\end{array}$ & $\begin{array}{l}\text { O Newborn Behavioral Observation ( } N B O \text { ) é uma alternativa clinicamente relevante } \\
\text { para a observação do recém-nascido na prática do visitante de saúde. O estudo é } \\
\text { baseado na comunidade, representando uma diversidade de novos pais e famílias que } \\
\text { recebem os serviços de saúde no período pós-natal. }\end{array}$ & $\mathrm{N} 2$ \\
\hline Cinhal & $\begin{array}{l}\text { Cava-Tadik/ } \\
\text { Journal of } \\
\text { Family Issues }\end{array}$ & EUA/ 2020 & $\begin{array}{l}\text { Durante o puerpério, alguns homens se sentem prejudicados em relação as tarefas } \\
\text { domésticas. Casais que dialogam sobre a distribuição das tarefas domésticas e os } \\
\text { cuidados com o bebê, tem mais afeto físico, enquanto pais que não acordam sobre } \\
\text { esta questão tem menos afeto físico e sexual. }\end{array}$ & $\mathrm{N} 2$ \\
\hline
\end{tabular}

Fonte: Autores (2020).

\section{Discussão}

Com a transição para maternidade/paternidade, a família se torna um grupo constituído por três seres, o que a transforma em um sistema permanente, pois mesmo que a união conjugal acabe o sistema sobrevive. Nesta lógica, simbolicamente e na realidade, tal transição constitui-se como transição-chave no ciclo de vida familiar (Carter \& McGoldrick, 2011).

Assim, constituir uma família com ramificações (filhos) requer que os adultos avancem uma geração para tornaremse cuidadores da geração mais jovem. Discussões e desentendimentos em torno desta questão ocorrem quando um dos pais não consegue realizar esta passagem (Carter \& McGoldrick, 2011).

Estudo americano analisado aponta que a chegada de um novo membro na família altera a dinâmica familiar, pois, a transição para maternidade/paternidade requer a reorganização na dinâmica e no relacionamento entre o casal, incluindo a relação conjugal, a educação dos filhos, a coparentalidade, a divisão do trabalho e o afeto (Cava-Tadik, et al., 2020).

Corroborando com este achado, outros autores afirmam que na atual sociedade na qual ambos cônjuges trabalham e tem carreiras, as relações familiares tendem a piorar pois surgem conflitos em torno, por exemplo, de tarefas domésticas, principalmente se ambos os pais trabalham em tempo integral (Carter \& McGoldrick, 2011). Além disso, são poucos os casais que partilham igualmente as tarefas domésticas e as responsabilidades pelos cuidados dos filhos (McGoldrick, 2011). Assim, em decorrência de uma provisão social insatisfatória há a tendência das diferenças aumentarem, a relação se desgastar e o casal acabar optando pela descontinuidade da relação conjugal (Carter \& McGoldrick, 2011).

Investigação assinala que após o parto as cargas de trabalho aumentam significativamente causando, às vezes, aumento no nível de estresse, especialmente para pais de primeira viagem (Maas, McDaniel, Feinberg, \& Jones, 2018). Ainda, o estresse associado a ter um bebê pode criar uma lacuna entre a proximidade dos casais e intimidade emocional, que são fatores essenciais relacionados com a relação sexual e a satisfação física (Radoš, Vraneš, \& Šunjić, 2015).

Neste sentido, muitos são os fatores que interferem negativamente no relacionamento conjugal entre os casais durante o puerpério. Alguns homens podem se sentir prejudicados, sobretudo, em relação as tarefas domésticas. Estudo americano incluído nesta revisão aponta que casais que dialogam sobre a distribuição das tarefas domésticas e os cuidados com o bebê tem mais afeto físico, enquanto pais que não acordam sobre a distribuição igualitária das funções tem menos afeto físico, sendo a vida sexual também impactada (Cava-Tadik, et al., 2020). Assim, parece que o estresse familiar é maior nos pontos de transição de um estágio para outro no processo de desenvolvimento familiar (Carter \& McGoldrick, 2011). 
Outro estudo americano incluído nesta revisão acerca do aumento da insatisfação conjugal e de conflitos após o nascimento de um filho, relata que a prática de exercício físico materno tem potencial para melhorar as relações familiares durante o puerpério, logo, pode ser um fator de proteção em situações em que as interações familiares são conflituosas ou estressantes (Hutt, et al., 2017). Acrescenta-se que a prática de exercícios físicos, desde que não contraindicada, além de melhorar as interações familiares, auxilia na recuperação física e psíquica da puérpera, pois, diminui o estresse, sintomas depressivos e fortalece o vínculo do binômio materno-infantil.

Outra estratégia, particularmente inovadora, destacada em estudo americano analisado corresponde a utilização de mídias sociais como suporte na passagem do puerpério, como mostra investigação americana realizada com mães afroamericanas e suas pessoas de apoio. Nesta pesquisa, meios digitais foram utilizados antes e após o parto para obter informações e compartilhar eventos de mudança de vida com familiares e amigos (Asiodu, et al., 2015).

A mesma investigação possibilitou o aprofundamento dos pesquisadores no que se refere aos comportamentos culturais, línguas, crenças, valores e costumes associados a alimentação infantil na comunidade afro-americana, grupo que historicamente não tem a amamentação como uma das suas práticas comuns. Com a chegada do bebê, houve mais interações presenciais entre pais, filhos e familiares, porém, algumas mulheres revelaram sentirem falta de apoio da família na travessia do período puerperal e minimizavam as suas contrariedades trocando experiências nas redes sociais com pessoas em situação semelhante (Asiodu, et al., 2015).

Sobre a falta de apoio da família, investigação brasileira analisada destaca a complexidade das dinâmicas familiares em face de um puerpério patológico. Estudo brasileiro informa que perturbações do humor como a DPP alteram os relacionamentos, pois provocam distanciamento conjugal pela falta de diálogo, carinho, amor e respeito por parte do parceiro e, no caso dos filhos, dificulta os cuidados com o recém-nascido e a interação mãe-filho. Assim, sentimentos negativos como culpa e medo são despertados na puérpera como consequências, piorando seu quadro depressivo (Melo, et al., 2015). Agravese a situação ao se considerar a atual sociedade na qual impera o discurso de maternidade dominante que criou relações binárias da "mãe boa" e "mãe má", fazendo com que as mulheres se sintam julgadas pela família e sociedade, como aponta investigação canadense selecionada (Aston, et al., 2015).

A DPP corresponde a um episódio de depressão temporalmente associado ao nascimento de um bebê cujo início, geralmente, se dá no final da gestação até cinco meses após o parto e cujo fim é imprevisto (Arrais \& Araújo, 2017). Neste sentido, é inegável a importância da atenção multiprofissional junto a mulher e sua família no puerpério acompanhado pelo adoecimento psicológico, todavia, nem sempre os profissionais encontram-se preparados para lidar com alterações do humor assim como a DPP (Gonçalves, et al, 2020).

Aliado aos profissionais, o cônjuge/marido, é uma figura importante neste processo para dar suporte à companheira e recém-nascido, como destacado em investigação brasileira analisada (Demarchi, et al., 2017). A família, também, é outra fonte de apoio considerada valiosa pelas puérperas, sendo a sua participação associada a melhorias na autoestima da mulher e como fonte de força para adaptação à nova realidade (Melo, et al., 2015; Demarchi, et al., 2017). O familismo, valor cultural significativo entre latinos e que se relaciona com a identificação e apego à família, se destacou em um dos estudos analisados de origem americana (Zeiders, et al., 2015).

Segundo tal investigação, os valores de familismo das avós, anterior ao nascimento do bebê, prediziam apoio e comunicação na criação dos filhos na díade avó-mãe adolescente após o nascimento do bebê. Neste sentido, o envolvimento intergeracional repercutiu positivamente nas decisões, da puérpera, que envolviam cuidados com o recém-nascido. Exercer o familismo suscitou comportamentos de apoio, sobretudo na criação da criança, ampliação da conexão e coesão familiar e redução dos conflitos conjugais, conforme mencionado em estudo americano analisado (Zeiders, et al., 2015). 
Nesta perspectiva, alerta-se que a definição de família, bem como o momento das fases do ciclo de vida e a importância concebida às distintas transições varia conforme o background cultural de cada grupo social, portanto, é fundamental analisar a questão da etnicidade e suas interferências no ciclo de vida para saber encorajar e orientar cada família (Carter \& McGoldrick, 2011).

Outra investigação incluída nesta revisão destaca a mãe da puérpera - figura feminina de referência cujo papel desempenhado de apoio diante das incertezas e dúvidas próprias ou não do período pós-parto é imprescindível (Melo, et al., 2015). Provavelmente, este achado tenha relação com a construção histórica e social que confere as mulheres a responsabilidade pela manutenção dos relacionamentos familiares e cuidados com marido, filhos, pais idosos, pais do cônjuge e qualquer outro membro da família doente ou dependente. Nada obstante, vale salientar que em nenhum momento da história a sociedade preocupou-se em oferecer provisão a esta expectativa e que aquelas mulheres que por algum motivo escolhem ou não conseguem atender a estas exigências, sente-se culpadas e sofrem (McGoldrick, 2011).

Além do apoio familiar, nas produções analisadas é citada a atuação dos profissionais de saúde durante o puérperio. Pesquisa canadense destaca a visita domiciliar realizada por enfermeiros de saúde pública, uma estratégia utilizada pelo governo deste país como forma de apoiar novas mães e suas famílias, sobretudo as consideradas vulneráveis. Durante as visitas domiciliares, os enfermeiros buscam estabelecer relações terapêuticas com as famílias com foco nos pontos fortes deste grupo e, a fim de subsidiar teoricamente as suas práticas, utilizam instrumentos tais como o MCAF e o MCIF (Aston, et al., 2015).

O MCAF surgiu em 1984 e consiste em um modelo abrangente e inclusivo que possibilita avaliar a família enquanto as enfermeiras discutem os problemas com seus membros. Assim, é capaz de fornecer uma macroavaliação ou "mapa da família" (considerando seus pontos fortes e problemas), até uma microavaliação a partir do exame de áreas específicas de funcionamento familiar. Já o MCIF, é uma estrutura organizadora para conceitualizar a interseção específica entre um domínio particular do funcionamento familiar e a intervenção proposta pela enfermeira, dando suporte à identificação do domínio familiar que necessita de mudança e qual intervenção é mais pertinente (Wright \& Leahey, 2018).

Considera-se que modelos como o MCAF e o MCIF contribuem para o planejamento e a gestão do cuidado de enfermagem e são ferramentas importantes para as enfermeiras da prática e da pesquisa construírem relacionamentos terapêuticos com seus pacientes. Relacionamentos terapêuticos nascem da boa comunicação, empatia, respeito e manutenção de interações recíprocas entre profissional e paciente.

Tais modelos, ao serem utilizados, permitem os profissionais acompanharem as mudanças nos primeiros dias, semanas, meses e até anos que possa durar o puerpério. É fundamental apoiar aquelas enfermeiras que desejam aprofundar-se na complexidade que envolve avaliar e intervir junto às famílias, não apenas para melhoria das práticas de saúde, o que sem dúvidas já seria inquestionavelmente justificável, mas para o bem-estar e a satisfação da família - foco do cuidado de enfermagem.

Pesquisa dinamarquesa analisada apresenta o Newborn Behavioral Observation (NBO), instrumento elaborado para descrever as competências do recém-nascido. Seu objetivo principal é fortalecer a relação entre pais e filhos e ajudar no desenvolvimento da relação entre profissionais e famílias. Em seus resultados indica que o $N B O$ é uma alternativa clinicamente relevante nas práticas dos visitantes de saúde quando se trata da observação das potencialidades e individualidades do recémnascido. Ainda, mediante um método cientificamente comprovado, possibilita reafirmar a importância da interação precoce saudável entre pais e bebês no puerpério, corroborando para a prática baseada em evidências (Kristensen \& Kronborg, 2018). 


\section{Considerações Finais}

As mudanças no ciclo de vida, embora importantes para o desenvolvimento familiar, provocam estresse e conflitos, muitas vezes, de difícil resolutividade. No caso do puerpério, é marcado por transformações que ultrapassam o binômio materno-infantil, afetando a dinâmica familiar. O apoio dos profissionais de saúde neste período é fundamental, em que pese que a boa comunicação junto a família, contribui na construção de relacionamentos terapêuticos e na adesão às orientações propostas.

É essencial, também, que os profissionais considerem as interações familiares a partir da realidade como um todo e isto só é possível mediante a observação deste grupo social no contexto domiciliar, pois o puerpério fisiológico é experienciado a maior parte do tempo no domicílio. Observou-se nos estudos analisados que os autores restringiram o contexto domiciliar ao local de coleta de dados, reduzindo a real importância deste espaço privado, de habitação e intimidade das pessoas, permeado de valores, crenças e hábitos, onde as relações afetivas e de cuidado acontecem. Assim, recomenda-se novas investigações com a temática, mas considerando o contexto domiciliar para que novos elementos sejam revelados e a lacuna de produção do conhecimento, até então existente, passe a ser preenchida.

Como limitação, tem-se que apenas algumas bases de dados foram consultadas e, embora intencional, somente artigos recentes e primários foram considerados na seleção. Todavia, a intenção das autoras foi suscitar novos questionamentos para mais investigações sobre a temática, dada a sua relevância e a necessidade de pesquisas envolvendo enfermagem e família.

\section{Agradecimentos}

À Coordenação de Aperfeiçoamento de Pessoal de Nível Superior (CAPES), pela concessão de bolsa de doutorado a uma das autoras.

\section{Referências}

Arrais, A. R., \& Araújo, T. C. C. F. (2017). Depressão pós-parto: uma revisão sobre fatores de risco e de proteção. Psic., Saúde \& Doenças, 18(3), 828-845. $10.15309 / 17$ psd180316

Asiodu, I. V., Waters, C. M., Dailey, D. E., Lee, K. A., \& Lyndon, A. (2015). Breastfeeding and use of social media among first-time african american mothers. J Obstet Gynecol Neonatal Nurs, 44(2), 268-278. 10.1111\%2F1552-6909.12552

Aston, M., Sheri, P., Etowa, J., Vukic, A., Young, L., Hart, C, MacLeod, E., \& Randel, P. (2015). The power of relationships: exploring how public health nurses support mothers and families during postpartum home visits. J Fam Nurs, 21(1), 11-34. 10.1177/1074840714561524

Brasil, Ministério da Saúde. (2016). Protocolos da Atenção Básica: Saúde das mulheres. http://bvsms.saude.gov.br/bvs/publicacoes/protocolos_atencao_basica_saude_mulheres.pdf

Brasil, Ministério da Saúde. (2011). Portaria n. 1.459, de 24 de junho de 2011. (2011). Institui no âmbito do Sistema Único de Saúde (SUS) a Rede Cegonha. http://bvsms.saude.gov.br/bvs/saudelegis/gm/2011/prt1459_24_06_2011.html

Carter, B., \& McGoldrick, M. (2011). As mudanças no ciclo de vida familiar: Uma estrutura para a terapia familiar. In Carter, B., \& McGoldrick, M. (Org.), As mudanças no ciclo de vida familiar: Uma estrutura para a terapia familiar (pp. 7-28). Artmed.

Cava-Tadik, Y., Brown, G. L., \& Mangelsdorf, S. C. (2020). Fathers' satisfaction with physical affection before and after the birth of a new baby: cross-parent effects and associations with family dynamics. Journal of Family Issues, 41(4). 10.1177\%2F0192513X19875779

Demarchi, R. F., Nascimento, V. F., Borges, A. P., Terças, A. C. P., Grein, T. A. D., \& Baggio, E. (2017). Perception of pregnant women and primiparous puerperas on maternity. Rev enferm UFPE online, 11(7), 2663-2673. 10.5205/1981-8963-v11i7a23438p2663-2673-2017

Gomes, I. M. (2013). A vivência do apoio da rede social pelas pessoas envolvidas no cuidado domiciliar. Dissertação de mestrado, Universidade Federal do Paraná, Curitiba, PR, Brasil

Gonçalves, C. L. S., Oliveira, A. M. N., Jantara, R. D., Gomes, J. C., Silva, M. R. S., \& Afonso, M. S. (2020) Conhecimento de profissionais da estratégia saúde da família acerca da depressão pós-parto. Research, Society and Development, 9(7), e337973842. 10.33448/rsd-v9i7.3842

Hutt, R. L., Moore, G. A., Mammen, M. A., \& Downs, D. S. (2017). Postpartum mothers' leisure-time exercise behavior is linked to positive emotion during partner discussions. Research Quarterly for Exercise and Sport, 88(4), 447-454. 10.1080/02701367.2017.1375450 
Kristensen, H. I., \& Kronborg, H. (2018). What are the effects of supporting early parenting by enhancing parents' understanding of the infant? Study protocol for a cluster-randomized community-based trial of the Newborn Behavioral Observation (NBO) method. BMC Public Health, 18, 832, 1-9. $10.1186 \%$ 2Fs40359-020-00467-5

Lockwood, C., Munn, Z., \& Porritt, K. (2015). Qualitative research synthesis: methodological guidance for systematic reviewers utilizing meta-aggregation. Int J Evid Based Healthc, 13(3), 179-187. https://journals.1ww.com/ijebh/Fulltext/2015/09000/Qualitative_research_synthesis_methodological.10.aspx

Maas, M. K., McDaniel, B. T., Feinberg, M. E., \& Jones, D. E. (2018). Division of labor and multiple domains of sexual satisfaction among first-time parents. Journal of Family Issues, 39(1), 104-27. 10.1177\%2F0192513X15604343

McGoldrick, M. (2011). As mulheres e o ciclo de vida familiar. In Carter, B., \& McGoldrick, M. (Org.), As mudanças no ciclo de vida familiar: Uma estrutura para a terapia familiar (pp. 29-64). Artmed.

Melo, W. S., Calderon, C. J., Monteiro, F. P. M., \& Veríssimo, F. A. S. (2015). Family relationship, needs and social life of woman with postpartum depression. Rev enferm UFPE on line, 9(3), 7065-1070. 10.5205/1981-8963-v9i3a10435p7065-7070-2015

Melnyk, B. M., \& Fineout-Overholt, E. (2005). Making the case for evidence-based practice. In Melnyk, B. M., \& Fineout-Overholt, E. (Org.), Evidence based practice in nursing \& healthcare: A guide to best practice (pp. 3-24). Lippincot Williams \& Wilkins.

Mendes, K. D. S., Silveira, R. C. C. P., \& Galvão, M. C. (2019). Uso de gerenciador de referências bibliográficas na seleção dos estudos primários em revisão integrativa. Texto contexto - enferm, 28(e20170204). 10.1590/1980-265x-tce-2017-0204

Montenegro, C. A. B., \& Rezende Filho, J. (2017). Obstetrícia fundamental (13a ed). Guanabara Koogan.

Organização Pan-Americana da Saúde. Organização Mundial da Saúde. (2018). Folha informativa: Mortalidade materna. https://www.paho.org/bra/index.php?option=com_content\&view=article\&id=5741:folha-informativa-mortalidade-materna\&Itemid=820

Paula, C. C., Padoin, S. S. M., \& Galvão, M. C. (2016). Revisão integrativa como ferramenta para tomada de decisão na prática clínica. In: Lacerda, M. M., Costenaro, R. G. S. (Org.) Metodologias da pesquisa para a enfermagem e saúde: Da teoria à prática (51-76). Moriá.

Radoš, S. N., Vraneš, H. S., \& Šunjić, M. (2015). Sexuality during pregnancy: what is important for sexual satisfaction in expectant fathers? Journal of Sex \& Marital Therapy, 41(3), 282-293. 10.1080/0092623x.2014.889054

Roma, J. C. (2019). Os objetivos de desenvolvimento do milênio e sua transição para os objetivos de desenvolvimento sustentável. Cienc. Cult, 71(1), 33-39. $10.21800 / 2317-66602019000100011$

Souza, J. P. (2015). A mortalidade materna e os novos objetivos de desenvolvimento sustentável (2016-2030). Revista Brasileira de Ginecologia e Obstetrícia, 37 (12), 549-551. 10.1590/SO100-720320150005526

Wright, 1. M., \& Leahey, M. (2018). Enfermeiras e famílias: Guia para avaliação e intervenção na família (5a ed). Roca.

Zeiders, K. H., Umaña-Taylor, A. J., Jahromi, L. B., \& Updegraff, K. A (2015). Grandmothers' familism values, adolescent mothers' parenting efficacy, and children's well-being. J Fam Psychol, 29(4), 624-34. 10.1037\%2Ffam0000103 